\title{
JD9
}

\section{Cold Gas and Dust at High Redshift}

Chairperson \& Editor: D.J. Wilner 


\title{
A Joint Discussion on the Topic of Cold Gas and Dust at High Redshift
}

\author{
D.J. Wilner \\ Harvard-Smithsonian Center for Astrophysics, 60 Garden St., \\ Cambridge, MA 02138, USA
}

The number of spectroscopically confirmed galaxies at high redshift is increasing rapidly, with many being found efficiently by deep optical imaging and color selection. In parallel, a confluence of technical developments is bringing rapid progress to the domain of observations of cold gas and dust at high redshift. Large telescopes operating at high, dry sites, with a new generation of sensitive detectors, together with recent satellite missions, are opening up new areas of study through observations of dust continuum emission and associated atomic and molecular emission and absorption lines. These data bear directly on fundamental questions of cosmic evolution by probing the ordinary cool material that forms stars and fuels active galactic nuclei. Analysis of data from the $C O B E$ satellite confirms the presence of a diffuse far-infrared background from a widespread population of distant dusty objects. The global energetics of the optical and far-infrared backgrounds suggest that perhaps half of distant activity may be enshrouded by dust. Understanding the nature and redshifts of the sources responsible for these emissions is profoundly important. The intent of Joint Discussion 9, between Division X (Radio Astronomy) and Division XIII (Galaxies and the Universe), was to provide a forum to present observations from this newly accessible realm and to consider the astrophysical implications.

Joint Discussion 9 at the XXXIVth IAU General Assembly, in Manchester, England, took place on August 14, 2000. A series of eleven speakers reviewed the following key scientific themes: Origin of Dust and Evolution of Galaxies (Fall, Andreani), Atomic Gas at High Redshift (Briggs), Far-Infrared, Submilleter and Millimeter Surveys of Dust Content at High Redshift (Elbaz, Barger, Bertoldi), Molecular Gas in Emission and Absorption at High Redshift (Carilli), The Optical Perspective on Hidden Star Formation (Meurer), Gas and Dust in Ultraluminous Galaxies (Downes), and Future Prospects (Kawabe, Blain). The day closed with a lively open discussion from all participants on controversial issues. We hope these discussions have helped to set the stage for advances expected from the many international instruments now under construction, including the Submillimeter Array, the Large Millimeter Telescope, the Atacama Large Millimeter Array, and the SIRTF, FIRST, and PLANCK missions.

Acknowledgments. I thank James Moran for the initial suggestion to organize this session, the JD9 scientific organizing committee for selecting excellent speakers, the many participants for their timely contributions, and the IAU for support. 